\title{
The Cytoskeleton Coordinates the Early Events of B-cell Activation
}

\author{
Naomi E. Harwood and Facundo D. Batista \\ Lymphocyte Interaction Laboratory, Cancer Research UK London Research Institute, Lincoln's Inn Fields \\ Laboratories, London WC2A 3LY, United Kingdom \\ Correspondence: facundo.batista@cancer.org.uk
}

B cells contribute to protective adaptive immune responses through generation of antibodies and long-lived memory cells, following engagement of the B-cell receptor (BCR) with specific antigen. Recent imaging investigations have offered novel insights into the ensuing molecular and cellular events underlying B-cell activation. Following engagement with antigen, BCR microclusters form and act as sites of active signaling through the recruitment of intracellular signaling molecules and adaptors. Signaling through these "microsignalosomes" is propagated and enhanced through B-cell spreading in a CD19-dependent manner. Subsequently, the mature immunological synapse is formed, and functions as a platform for antigen internalization, enabling the antigen presentation to helper $\mathrm{T}$ cells required for maximal B-cell activation. In this review, we discuss the emerging and critical role for the cytoskeleton in the coordination and regulation of these molecular events during B-cell activation.

\begin{abstract}
$\mathrm{A}^{\mathrm{n}}$ individual is continually bombarded by Apotentially disease-causing agents. However, under normal circumstances, the action of the immune system ensures that these encounters relatively infrequently result in the development of symptomatic illness. The immune system can be broadly divided into two component parts, the innate and the adaptive branches. The innate branch mediates rapid inflammatory responses following the recognition of motifs typically associated with pathogens through a collection of pattern recognition receptors. In contrast, adaptive immune responses tend to emerge a few days after initial infection and show four central characteristics: memory, specificity, diversity, and self-nonself discrimination. In spite of these two divisions, the effective elimination
\end{abstract}

of pathogens most often requires intricate collaboration between the innate and adaptive immune responses.

Small white blood cells, known as lymphocytes, are the fundamental participants mediating adaptive immune responses. Lymphocytes originate from hematopoietic stem cells in the bone marrow and although a subset migrate to the thymus to form $\mathrm{T}$ cells, others remain in the bone marrow to complete their development into B cells (Halin et al. 2005). Mature lymphocytes circulate throughout the body and are often localized in secondary lymphoid organs such as the lymph nodes and the spleen, which are specialized sites for lymphocyte activation (Junt et al. 2008). T cells are responsible for cell-mediated immunity and are commonly

Editors: Lawrence E. Samelson and Andrey S. Shaw

Additional Perspectives on Immunoreceptor Signaling available at www.cshperspectives.org

Copyright (C) 2011 Cold Spring Harbor Laboratory Press; all rights reserved; doi: 10.1101/cshperspect.a002360

Cite this article as Cold Spring Harb Perspect Biol 2011;3:a002360 
classified into $\mathrm{CD}^{+}$helper and $\mathrm{CD}^{+}$cytotoxic cells according to their expression of surface coreceptors. On the other hand, B cells mediate humoral immunity through the secretion of antibodies that recognize and neutralize invading pathogens.

To become activated to produce antibodies, $B$ cells must first recognize specific antigen through the B-cell receptor (BCR). This specific binding event initiates intracellular signaling leading to altered gene expression, reorganization of the B-cell cytoskeleton, and antigen internalization. Importantly, BCR-mediated internalization targets antigen to endosomes containing newly synthesized major histocompatibility complex (MHC) (Aluvihare et al. 1997; Amigorena et al. 1994), such that processed antigen can be presented to $\mathrm{CD}^{+}{ }^{+} \mathrm{T}$ cells, thereby recruiting help to facilitate maximal B-cell activation (Lanzavecchia 1985; Rock et al. 1984). Activated B cells can either rapidly mediate the secretion of low affinity antibodies (MacLennan et al. 2003), or can enter into a specialized structure known as a germinal center (GC) to undergo affinity maturation, producing plasma cells capable of high-affinity antibody production and long-lasting memory cells (MacLennan 1994; Rajewsky 1996).

The molecular events underlying BCR-mediated signaling have historically been characterized using standard biochemical analysis methods in vitro. As such, the BCR has been identified as comprising of a membrane immunoglobulin $(\mathrm{mIg})$ responsible for binding extracellular antigen, in complex with an $\operatorname{Ig} \alpha /$ $\beta$ sheath containing immunoreceptor tyrosine activation motifs (ITAMs) in the intracellular domains (Reth 1989). Cross-linking of the BCR by multivalent antigen triggers phosphorylation of the ITAMs through Src family kinases such as Lyn and Syk. This early phosphorylation leads to the recruitment of intracellular effectors including PLC $\gamma 2$, Vav, Btk, and PI3K, and adaptors including Blnk and Grb2, to form a multicomponent assembly known as the signalosome (Dal Porto et al. 2004; DeFranco 1997; Kurosaki 2002; Scharenberg et al. 2007). Cellular readouts of the coordinated activity of the signalosome include calcium signaling and activation of transcription factors such as NF- $\mathrm{kB}$. Although these classical strategies have provided an essential foundation of intracellular mediators involved in signaling downstream of the BCR, they contribute little insight into the spatiotemporal dynamics and organization of molecular events within the cell (Treanor and Batista 2007).

To address the questions of how and where molecular events occur within the cell, imaging-based strategies have been developed and applied to visualize directly molecular events underlying B-cell activation. Importantly, these approaches not only allow verification of molecular pathways identified previously, but also shed light on the spatiotemporal dynamics of these pathways within the cell. In addition, imaging approaches have led to the identification of unexpected and novel roles for molecules such as CD19 in mounting B-cell responses to antigen. An essential tenet that has been revealed by these imaging studies is the importance of molecular segregation as a means of coordinating and regulating interactions within the cell. Indeed, a pivotal role for the cytoskeleton as a means of mediating segregation is emerging, offering a versatile mechanism for the regulation of numerous molecular interactions. The importance of the cytoskeleton is evidenced by the recent observation that mutations in various cytoskeleton regulators are associated with the development of antibody deficiency syndromes in humans (Conley et al. 2009). In this review, we discuss the contribution that recent imaging investigations have made to our understanding of the process and regulation of B-cell activation. In particular, we highlight the central role for the cytoskeleton in the organization and control of molecular events during B-cell activation.

\section{BCR DISTRIBUTION IN THE RESTING B CELL IS SHAPED BY THE CYTOSKELETON}

As activation is initiated following cross-linking of the BCR by antigen, it is important to characterize the distribution and dynamics of the BCR in the resting state to fully appreciate the molecular events regulating the initiation of B-cell activation. In view of the fact that multivalent, 
but not monovalent, soluble antigens trigger activation (S. Minguet, M. Reth, W. Schamel, pers. comm.; Tolar et al. 2009), it was widely assumed that the BCR exists as a monomer in resting $\mathrm{B}$ cells. However, as native gel electrophoresis showed that BCRs are able to interact with one another, it was suggested that BCRs form oligomers on the surface of the resting B cell (Schamel and Reth 2000). On the contrary, a fluorescence resonance energy transfer approach was unable to detect an interaction between individual BCRs in the membrane, indicating that BCRs are monomeric in the absence of antigen (Tolar et al. 2005). Thus, the precise state of the BCR in the membrane before antigen stimulation remains controversial.

Recently, we have sought to visualize the dynamics of BCR on the surface of the resting B cells using Dual-View total internal reflection microscopy (TIRFM) (Treanor et al. 2010) (Fig. 1A). Using this method, we were able to track single particles of $\mathrm{BCR}$ in the resting $\mathrm{B}$-cell membrane. We observed that BCRs were not able to diffuse freely in the plane of the membrane and instead show a range of diffusion behaviors, with a proportion of receptors much less mobile (diffusion coefficient less than $<0.01 \mathrm{um}^{2} \mathrm{~s}^{-1}$ ). This restriction in diffusion is contrary to the predictions of the original fluid-mosaic model proposed by Singer and Nicholson (1972); however, it is in line with more recent investigations tracking single molecules of transmembrane proteins in fibroblasts (Kusumi et al. 2005; Sako and Kusumi 1994; Simson et al. 1995). Indeed, these observations led the authors to suggest that the cell membrane contains discrete confinement zones around $30-700 \mathrm{~nm}$ in diameter that restrict long-range diffusion between adjacent zones. This is known as the "picket-fence" model, in which selected transmembrane proteins act as pickets defining confinement zones and are attached to the underlying cytoskeleton fence (Fujiwara et al. 2002). Furthermore, it has
A

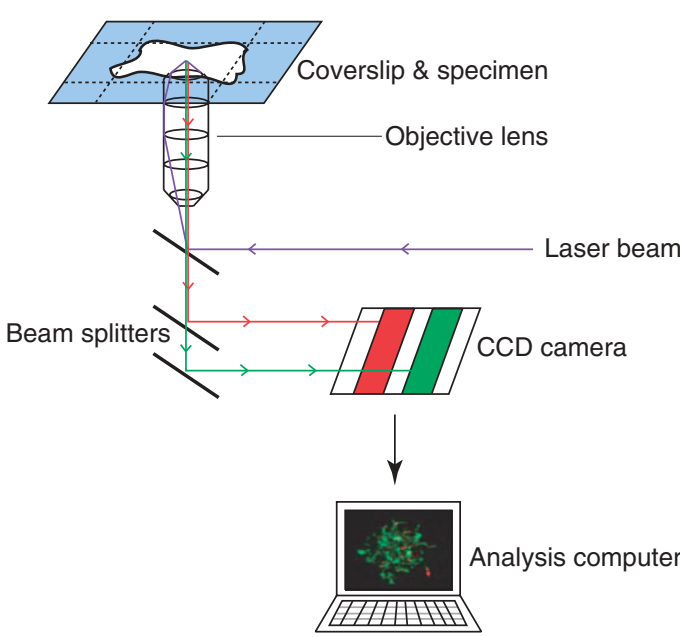

B
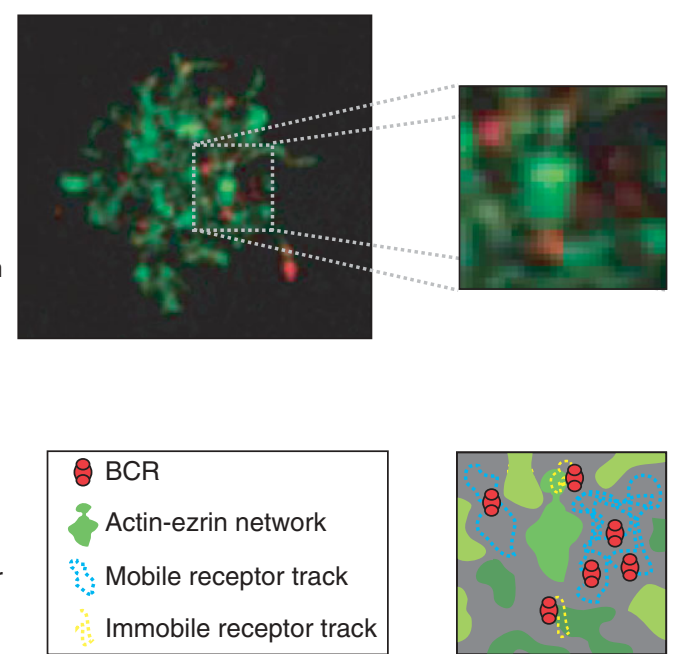

Figure 1. Dual-View TIRFM for simultaneous tracking of BCR alongside the actin cytoskeleton. (A) Schematic representation of the setup of the Dual-View TIRFM. The total internal reflection of incoming laser beam (purple) generates evanescent waves capable of exciting flurophores in close proximity to the sample interface. The two colored emission beams (green and red) are passed through beam splitters, detected simultaneously by a CCD camera and overlaid in an analysis computer. (B) Snapshot of a movie collected tracking single molecules of BCR (red, anti-BCR Fab) and actin (green, LifeAct a marker of filamentous actin). BCRs located in actin-rich areas are more restricted in their diffusion (yellow tracks in schematic zoom), whereas BCRs located in actin-poor areas tend to be less restricted in their diffusion (blue tracks in schematic zoom). 
been suggested that lipid microdomains and/or protein islands may contribute to the observed restriction in diffusion of transmembrane proteins (Lillemeier et al. 2006; Simons and Ikonen 1997). Interestingly, we have used our methodology to simultaneously visualize the BCR alongside components of the cytoskeleton, and showed that slow-moving or immobile BCRs were often co-incident with regions with high density of an ezrin-defined actin network (Treanor et al. 2010) (Fig. 1B). Indeed, disruption of the cytoskeleton network through pharmacological agents results in intracellular signaling similar to that observed following antigen stimulation, likely as a result of BCR cluster formation. Thus, it appears that the cytoskeleton plays an important role in regulating the distribution and dynamics of the BCR in the resting B cell.

\section{RECOGNITION OF ANTIGEN TRIGGERS B-CELL ACTIVATION BY AN UNKNOWN MECHANISM}

The BCR can recognize and respond to native antigens both in soluble or membrane-bound forms, though the latter have a lower threshold and thus represent a more effective means of triggering B-cell activation (Batista et al. 2001; Batista and Neuberger 1998, 2000). Although it has been known for some time that dendritic cells (Huang et al. 2005; Wykes et al. 1998), follicular dendritic cells (Chen et al. 1978; Mandel et al. 1980; Tew et al. 1980), and macrophages (Koppel et al. 2005) can present intact antigen to $B$ cells in vitro, very recent multiphoton microscopy investigations have verified the physiological significance of antigen on the surface of presenting cells in mediating B-cell activation in vivo (Carrasco and Batista 2007; Junt et al. 2007; Phan et al. 2007; Phan et al. 2009; Qi et al. 2006; Suzuki et al. 2009). In view of the predominance of membrane-bound antigen in initiating activation, the original hypotheses developed to describe BCR triggering in response to soluble antigen stimulation must be re-examined. Indeed, it has been observed that monovalent antigen attached to the surface of a presenting cell, unlike monovalent soluble antigen, can trigger B-cell activation (Tolar et al. 2009). Furthermore, the additional constraints that are imposed on the B cell to recognize antigen immobilized on the surface of a presenting cell must be considered. As T cells absolutely require the recognition of antigen in complex with MHC on the surface of a presenting cell, models developed to describe the initiation of T-cell activation may prove useful in the derivation of a general description of activation of B lymphocytes.

As the modules responsible for ligand binding and intracellular signaling in immunoreceptors such as the BCR and TCR are separated, the molecular mechanism by which the external binding of antigen is communicated across the membrane is not immediately obvious. Indeed, this has been the subject of a number of investigations and still remains controversial. Two most likely models have emerged to explain the triggering of activation in T cells (Fig. 2). The first of these is perhaps the more simple conceptually and proposes that the binding of antigen to the extracellular region is communicated to the intracellular signaling region through conformational change(s) in the intervening domains (Kuhns et al. 2006; Schamel et al. 2006) (Fig. 2A). Although high-resolution static crystallographic data have not provided evidence in support of this view (reviewed in Garcia et al. 1999; Hennecke and Wiley 2001; Rudolph et al. 2006; van der Merwe 2001), a number of recent studies have identified conformational changes in the CD3 $\varepsilon$ domain of the TCR following ligation such that CD3e ITAM motifs are exposed to activating Src-family kinases and binding of intracellular effectors (Gil et al. 2002; Xu et al. 2008). The second model is known as kinetic-segregation and explains the initiation of T-cell triggering through the alterations in the membrane distribution of inhibitory phosphatases, such as CD45 (Choudhuri et al. 2005; Davis and van der Merwe 2006) (Fig. 2B). This model assumes that, before antigen stimulation, the TCR is at equilibrium such that it is equally likely to be phosphorylated or dephosphorylated. However, on contact with an antigen-presenting cell, small adhesion molecules such as $\mathrm{CD} 2$ on the T-cell surface 
A

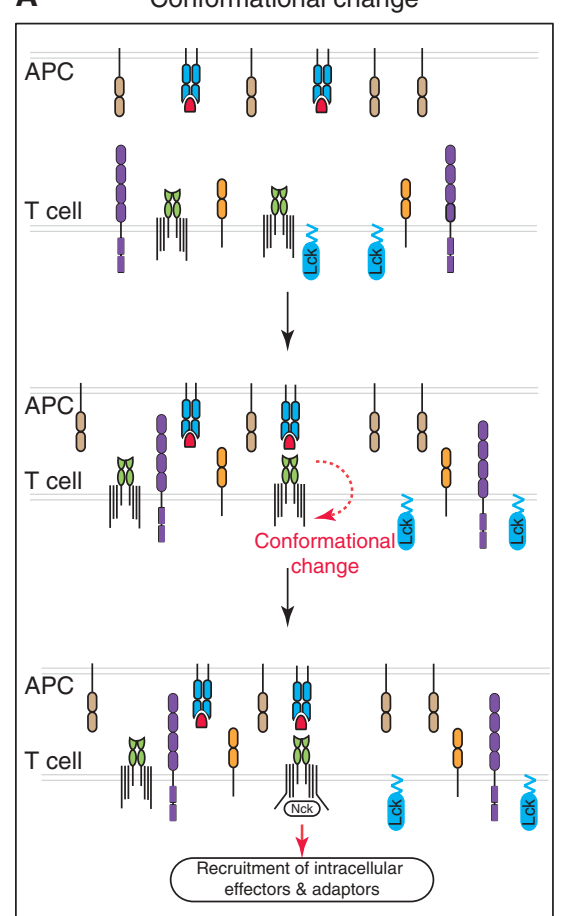

B

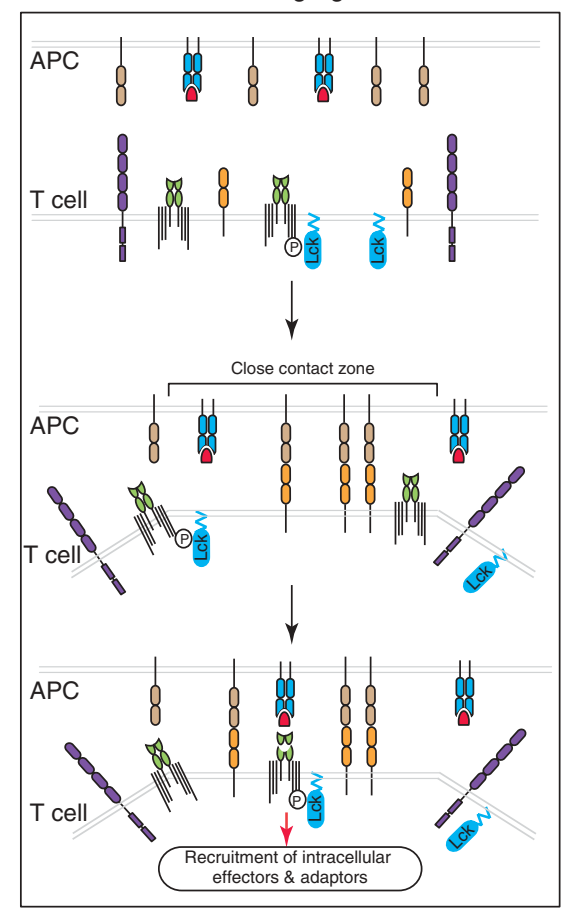

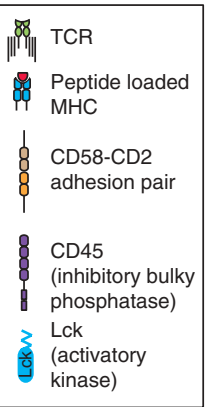

Figure 2. Two models of T-cell triggering. (A) The conformational change model states that extracellular pMHC binding to the TCR triggers intracellular conformational changes in the associated CD3 complex, opening up binding sites for the intracellular adaptor Nck. Subsequently, the "opened" TCR recruits activatory kinases (such as Lck) to phosphorylate the TCR, leading to assembly of the signalosome. (B) The kinetic segregation model postulates that, in the resting state, TCR is equally likely to be phosphorylated by activatory kinases or dephosphorylated inhibitory phosphatases. On proximity of an antigen-presenting cell, small adhesion molecules such as CD2 and CD58 establish a "close contact zone" that excludes bulky surface molecules such as CD45, but permits diffusion of TCR and Lck. TCR in the "close contact zone" is more likely to be found in a phosphorylated state, and the half-life of phosphorylated TCR in this region is increased following recognition of specific pMHC, triggering the assembly of the signalosome and T-cell activation.

establish close-contact zones that exclude more bulky cell-surface molecules, including CD45. Given that the extracellular domain of the TCR is relatively small, its diffusion into and out of the close contact zone is not restricted. Accordingly, TCR within the close contact zone is more likely to be phosphorylated because of the size-dependent exclusion of CD45. When the TCR residing in the close contact zone specifically recognizes antigen on the surface of the presenting cell, it will be held in this zone, increasing the lifetime of phosphorylated TCR, such that triggering occurs. There are a number of lines of experimental evidence supporting this viewpoint, including the importance of the balance of phosphorylation (Secrist et al. 1993) and the size-dependence of various extracellular domains for triggering T-cell activation (Choudhuri et al. 2005; Irles et al. 2003). Taken together, it seems likely that some features of both the conformational change and the kinetic-segregation models participate during the initiation of TCR triggering.

Can these two models prove useful in the description of analogous processes during B-cell activation? At this stage, there is no high-resolution structural data that supports a rapid conformational change to initiate triggering of the 
BCR following antigen binding; however, this does not necessarily eliminate the possibility that conformational changes may play a role in the initiation of BCR triggering. Indeed, quantitative FRET imaging has revealed conformational changes in the cytoplasmic domains of the BCR complex that are dependent on Lyn recruitment and activity, and thus are assumed to occur later on during BCR signaling (Tolar et al. 2005). In terms of the kineticsegregation model, it is clear that the balance of phosphorylation is important for the initiation of B-cell activation (Rolli et al. 2002). Interestingly, although CD45 is the predominant cellsurface phosphatase in T cells (Mustelin et al. 1989), B cells express significant amounts of another phosphatase CD148 (Zhu et al. 2008). Thus, though CD45-deficient B cells are not significantly impaired in their B-cell activation (Byth et al. 1996; Depoil et al. 2008; Kishihara et al. 1993), it is possible that the bulkier CD148 may play a redundant role in size-dependent exclusion during B-cell triggering. As such, it will be necessary to investigate $B$ cells deficient in CD45 and CD148 to assess the validity of the kinetic-segregation model to describe the initiation of BCR triggering. Future imaging investigations will provide insight into the dynamic distribution of various molecules in the B-cell membrane, and thus shed light on the precise mechanism underlying BCR triggering.

\section{BCR MICROCLUSTERS FORM AND MEDIATE INTRACELLULAR SIGNALING AFTER ANTIGEN STIMULATION}

Although imaging methods have not yet provided a definitive molecular mechanism underlying the initiation of BCR triggering, they have proved incredibly informative in the characterization of early events of B-cell activation. Currently, the earliest observable event associated with successful antigen stimulation is the formation of microclusters of BCR in the membrane (Fig. 3). BCR microclusters have been visualized using TIRFM and time-lapse wide-field fluorescence microscopy following settling of B cells on planar lipid bilayers containing antigen (Depoil et al. 2008; Fleire et al. 2006). These microclusters are formed of approximately 50 to 500 BCR molecules and can be comprised of either or both of the BCR istoypes expressed by primary B cells, IgM, and IgD (Depoil et al. 2008).

Interestingly, the formation of BCR microclusters was not impaired in Lyn-deficient B cells (Weber et al. 2008), indicating that this process is not dependent on signaling through the BCR. In contrast, the formation of BCR microclusters is absolutely dependent on alterations in the underlying cytoskeleton (Depoil et al. 2008). In line with this, we have very recently observed that the ezrin-actin network underlying the B-cell membrane is reorganized following antigen stimulation, such that defined "corrals" form around BCR microclusters (B. Treanor and F.D. Batista, unpubl. data). Furthermore, as these corrals restrict BCR diffusion, it seems likely that the cytoskeleton network may contribute to the stability and integrity of BCR microclusters. Thus, it could be that BCR microclusters form as a result of diffusion trapping following restriction of diffusion by the B-cell cytoskeleton. In addition, we expect that other factors play a role in formation of BCR microclusters. In line with this, Pierce and colleagues observed that the membrane-proximal $\mathrm{C} \mu 4$ domains of $\operatorname{IgM}$ have the propensity to oligomerize, suggesting that these domains play an important role in microcluster formation (Tolar et al. 2009). The authors do, however, note that in order to mediate this function, the IgM molecules must first be confined within the membrane, potentially through the action of the B-cell cytoskeleton.

The assembly of BCR microclusters was observed to occur on a timescale coincident with the initiation of calcium signaling, suggesting that they may play a role in mediating intracellular signaling. In line with this, early observations indicated that microclusters of antigen often were coincident with staining for phosphotyrosine (Fleire et al. 2006) and excluded inhibitory phosphatases such as CD45 (Depoil et al. 2008). We used an approach involving the expression of fluorescently labeled intracellular effectors in B cells and observed the impact of settling on antigen-containing lipid bilayers by 


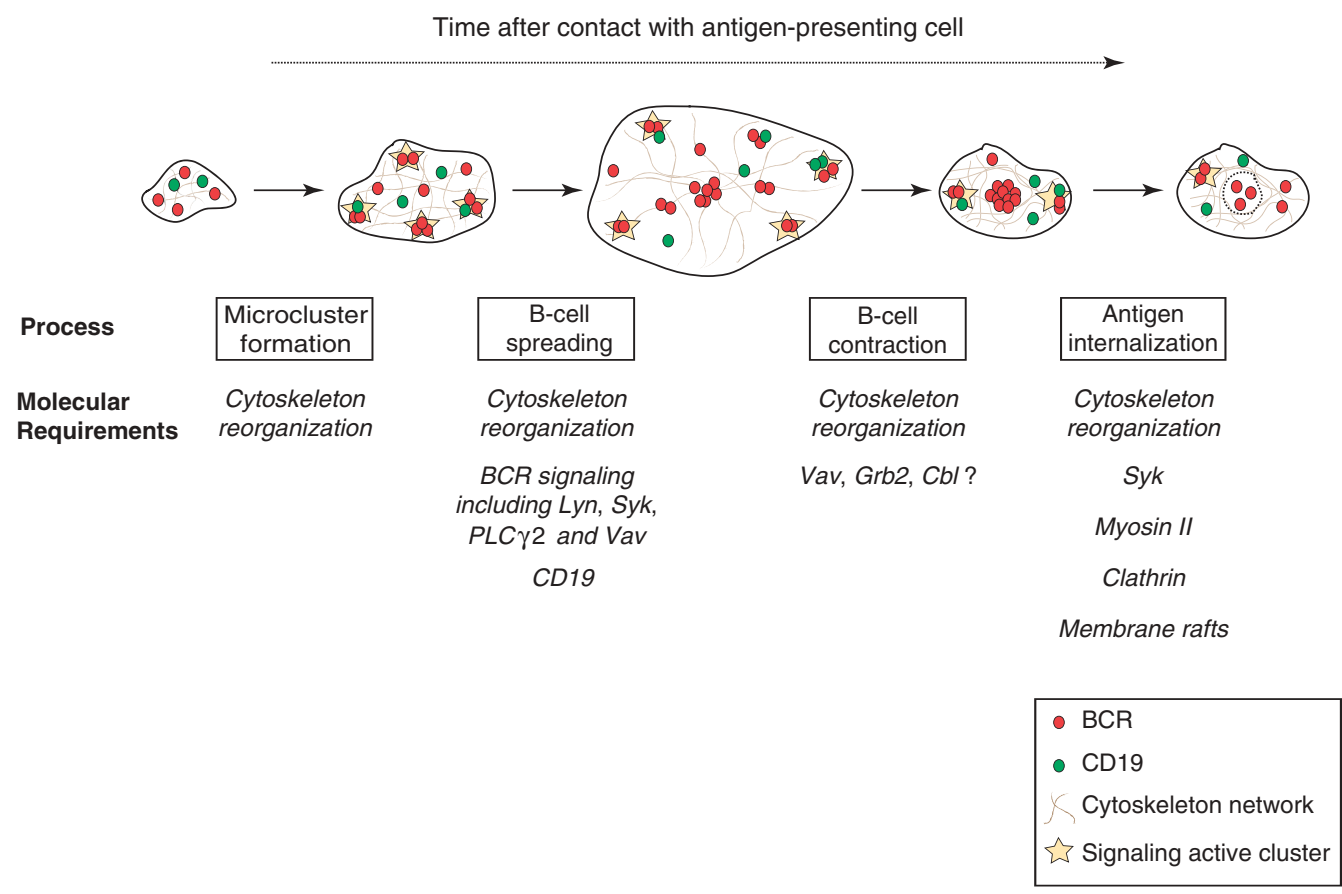

Figure 3. B-cell activation by antigen on the surface of a presenting cell. Schematic representation of the processes occurring at the B-cell surface following recognition of antigen on a presenting surface and what is currently known about the molecular requirements of these processes. On initial contact with antigen, BCR microclusters form, driving B-cell spreading. During spreading, signaling active BCR microclusters are moved (possibly via reterograde actin flow) toward the central cluster. Following achievement of maximum spread, the more prolonged contraction phase occurs, resulting in the formation of the mature immunological synapse. The central cluster of the immunological synapse acts as a platform for internalization of antigen. It appears that at every stage, cytoskeleton reorganizations play an important role in orchestrating and coordinating the molecular processes.

three-color TIRFM. In this way, we visualized the sequential and highly coordinated recruitment of the initiating kinases Lyn and Syk, followed by the downstream mediators Vav and PLC 22 (Weber et al. 2008). A subsequent FRETbased investigation confirmed the recruitment of Lyn to BCR microclusters and suggested that membrane lipids may play a role in the regulation of this association (Sohn et al. 2008). In addition, we also showed that not only the recruitment but also the enzymatic activity of particular mediators was required to initiate calcium signaling (Weber et al. 2008). These observations indicate that BCR microclusters function to mediate intracellular signaling. As this is in agreement with previous observations concerning the role of TCR microclusters
(Bunnell et al. 2002; Campi et al. 2005; Yokosuka et al. 2005), we have redefined microclusters as microsignalosomes and suggested that they are a common unit of signaling in lymphocytes (Depoil et al. 2008; Harwood and Batista 2008). Importantly, microsignalosomes allow intricate regulation of immunoreceptor signaling through the potential for dynamic recruitment of positive and negative regulators. In line with this, we have observed that components of microsignalosomes can cooperate to facilitate the recruitment and retention of the other (Weber et al. 2008). This type of elegant mechanism for regulating BCR signaling is not possible from "cap" structures previously observed following stimulation with soluble antigen (Schreiner et al. 1976; Stackpole et al. 
1974; Unanue et al. 1972). Thus, given that the predominant form of antigen-mediating activation in vivo is membrane-bound, $B$ cells can very precisely regulate the extent of activation according to the context in which the antigen is presented.

\section{SPREADING PROPAGATES MICROSIGNALOSOMES NECESSARY FOR B-CELL ACTIVATION}

Although microsignalosomes are required for mediating BCR signaling their formation alone, is not sufficient to elicit B-cell activation. In addition, it is necessary for the B-cell to amplify the number of microsignalosomes such that the threshold for antigen stimulation is exceeded (Weber et al. 2008). The B cell achieves this amplification by spreading out its membrane across the presenting cell, allowing contact with greater amounts of antigen on the surface (Fleire et al. 2006) (Fig. 3). As BCR engagement of antigen at the leading edge drives the spreading response, the extent of spreading depends on the affinity and density of antigen on the presenting surface and determines how much antigen the $\mathrm{B}$ cell accumulates for presentation to helper T cells (Fleire et al. 2006). As spreading is abrogated in the presence of inhibitors of actin polymerization and Src-family kinases, this cellular response absolutely requires intracellular signaling and cytoskeleton reorganization.

The signaling events downstream of the BCR important for mediating spreading have recently been investigated (Weber et al. 2008) through a screen of an existing panel of mutants in a chicken B-cell line (Shinohara and Kurosaki 2006). The DT40 B-cell line shows a high rate of homologous recombination, enabling the rapid generation of B cells deficient in a particular mediator (Kurosaki 1999). In addition, this strategy has the advantage of allowing investigation of molecules whose deletion is embryonically lethal in a knockout animal system. It was observed that Lyn and Syk were absolutely required for the initiation of B-cell spreading in response to antigen-containing membranes
(Weber et al. 2008). Furthermore, although B cells deficient in Vav and PLC 22 were severely impaired in mediating spreading, it is clear that these two mediators play a critical role in the propagation of the cellular response. B cells deficient in Btk and Blnk were markedly impaired in spreading, suggesting that the activation and retention of PLC $\gamma 2$ is important during spreading. In contrast, $\mathrm{B}$ cells lacking all three $\mathrm{IP}_{3}$ receptors were fully competent in mediating spreading, a somewhat surprising observation given the calcium-dependence of the analogous process in T cells (Bunnell et al. 2001).

Although the key intracellular mediators involved in B-cell spreading have been established, the molecular mechanisms underlying the necessary cytoskeleton rearrangements currently remain unclear. However, as Vav is known to be an important regulator of the cytoskeleton and has been established as critical for propagation of B-cell spreading (Weber et al. 2008), this offers insight into potential mechanisms for reorganization of the cytoskeleton. Alongside its role as a molecular adaptor, Vav functions as a guanine nucleotide exchange factor (GEF) to activate RhoGTPases such as $\mathrm{Cdc} 42$ and Rac that are involved in the regulation of cytoskeleton reorganizations (Jaffe and Hall 2005). In line with this, the GEF activity of Vav has been shown to be essential during B-cell spreading (Weber et al. 2008). In addition, in T cells, Vav has been implicated as a regulator of ezrin dephosphorylation in response to TCR stimulation (Faure et al. 2004). This is of particular interest as dephosphorylation of ezrin leads to release of the association between the plasma membrane and the actin cytoskeleton, facilitating the formation of cell conjugates. Interestingly, a rapid and global dephosphorylation of ezrin has been observed following stimulation of B cells with soluble antigen (Gupta et al. 2006). In conjunction with Vav, a number of other intracellular B-cell effectors have been implicated in the regulation of cytoskeleton reorganizations following stimulation of the BCR. These include the leukocyte-specific homolog of cortactin HS1 (Gomez et al. 2006; Hao et al. 2004; Yamanashi et al. 1997), cofilin 
(DesMarais et al. 2005; Yonezawa et al. 1991), DOCK8 (Randall et al. 2009), and B-lymphocyte adaptor molecule of $32 \mathrm{kDa}$ (Bam32) (Fournier et al. 2003; Han et al. 2003; Marshall et al. 2000). Bam32 represents a particularly interesting candidate as it is recruited to the plasma membrane and phosphorylated following BCR ligation (Marshall et al. 2000), and $B$ cells deficient in Bam32 are impaired in actin polymerization and antigen internalization (Niiro et al. 2004). Given the importance of the spreading response in shaping the outcome of B-cell activation, detailed investigations of these and other mediators will be invaluable in deriving a complete description of the molecular mechanism communicating BCR ligation with cytoskeleton reorganizations.

\section{CD19 IS ESSENTIAL FOR B-CELL SPREADING AND ACTIVATION}

During a dissection of the requirements for B-cell spreading, we uncovered a novel and unexpected role for the B-cell coreceptor CD19 (Depoil et al. 2008). Until this discovery, CD19 was most commonly known as a component of the CD21-CD19-CD81-leu13 complex responsible for enhanced BCR signaling in response to antigen coated with complement fragments (Fearon and Carroll 2000). In these circumstances, the ligation of CD21 (also known as complement receptor 2) triggers CD19 to recruit intracellular effectors that facilitate signaling through the BCR. However, the observation that CD19-deficient mice show more severely impaired B-cell responses to antigen than mice lacking CD21 (Ahearn et al. 1996; Engel et al. 1995; Rickert et al. 1995) suggests that CD19 performs an alternative role independent of CD21 during B-cell activation. In line with this, we observed that B cells lacking CD19 were unable to initiate calcium signaling and thus could not mediate spreading and activation in response to antigen on the surface of presenting cells (Depoil et al. 2008). Interestingly, and in agreement with previous investigations, we observed that CD19-deficient $B$ cells respond to soluble antigen in a similar manner to wild-type B cells (Depoil et al. 2008; Fujimoto et al. 1999; Sato et al. 1997). These observations nicely complement recent intravital imaging investigations, demonstrating the central role of antigen bound to presenting cells as the predominant form triggering B-cell activation in vivo (Carrasco and Batista 2007; Junt et al. 2007; Phan et al. 2007; Phan et al. 2009; Qi et al. 2006; Suzuki et al. 2009).

Though the molecular mechanism by which CD19 mediates this essential role in B-cell spreading has not yet been fully elucidated, imaging strategies have allowed us to gain some insight into this process. The cytoplasmic domain of CD19 contains several motifs that can mediate the recruitment of numerous intracellular effectors, including PI3K and Vav ( $\mathrm{Li}$ et al. 1997; Tuveson et al. 1993). Furthermore, it has been suggested that CD19 forms oligomers in the membrane (Brooks et al. 2004), potentially allowing cooperation and prolonged retention of recruited molecules. Thus, it seems plausible to suggest that CD19 mediates the recruitment and stabilization of additional intracellular effectors in the microsignalosome to facilitate signaling through the BCR. In support of this mechanism, it was observed that CD19 becomes transiently associated with BCR microclusters following stimulation with membrane-bound antigen (Depoil et al. 2008). More recently, we have used Dual-View TIRFM to visualize single particles of CD19 moving between signaling BCR microclusters following antigen stimulation (D. Depoil and F.D. Batista, unpubl. data). In addition, we observed that mediators recruited through CD19, such as PI3K, are laterally segregated from those recruited solely through the BCR in the B-cell membrane. Thus, at this stage, it appears that CD19 plays a role in B cells similar to that of LAT in T cells (Zhang and Samelson 2000), providing the boost to BCR signaling necessary to stimulate cytoskeleton reorganization. Interestingly, mutations in CD19 and other potential mediators of cytoskeleton reorganization have been associated with antibody deficiency syndromes in humans (Conley et al. 2009). 


\section{B-CELL CONTRACTION RESULTS IN FORMATION OF THE IMMUNOLOGICAL SYNAPSE}

B cells attain maximum spread a few minutes after initial contact with an antigen-presenting cell and subsequently the B-cell membrane undergoes a more prolonged contraction phase (Fleire et al. 2006) (Fig. 3). The mechanism underlying the initiation of B-cell contraction remains unclear; however, a genetic screen to identify mediators involved in the spreading response revealed that $\mathrm{B}$ cells deficient in Vav, Grb2, or Cbl showed impaired contraction (Weber et al. 2008; T. Schnyder and F.D. Batista, unpubl. data). B-cell contraction results in the dramatic reorganization of the membrane forming a structure known as the immunological synapse (IS) (Batista et al. 2001; Carrasco et al. 2004). The IS was originally identified in $\mathrm{CD}^{+}{ }^{+} \mathrm{T}$ cells (Grakoui et al. 1999; Krummel et al. 2000; Monks et al. 1998) and has since become recognized as a common feature associated with lymphocyte activation (Davis et al. 1999; Potter et al. 2001; Stinchcombe et al. 2001). The IS comprises a cluster of immunoreceptors in a central supramolecular activation cluster (cSMAC) surrounded by a ring of adhesion molecules, such as the integrins LFA-1 and VLA-4, in the peripheral SMAC (pSMAC) (Monks et al. 1998). It is thought that during spreading, clusters of signaling immunoreceptors associated with the underlying actin cytoskeleton are passively translocated to the center of contact via retrograde actin flow to assemble the cSMAC (Kaizuka et al. 2007). Interestingly, this would provide an elegant mechanism to explain the segregation of molecules within the IS according to their particular association with components of the cytoskeleton. In addition, recent data suggested that in $\mathrm{T}$ cells, this transportation is also mediated through an active process involving the actin-based molecular motor myosin IIA (Ilani et al. 2009). However, the contribution of the two mechanisms or alternatives during contraction and formation of the IS in B cells remains to be established.

Though imaging was used to visualize the structure more than a decade ago, the function of the IS has remained controversial. It was originally put forward that given the concentration of immunoreceptors in the CSMAC, this would likely represent the site for active signaling. However, this assumption must be revisited given that intracellular calcium signaling is observed more rapidly than the formation of the mature IS, and in view of the more recent observation that microclusters of immunoreceptors actively signal the cell periphery during spreading (Bunnell et al. 2002; Campi et al. 2005; Depoil et al. 2008; Weber et al. 2008; Yokosuka et al. 2005). In line with this, a recent investigation tracking microcluster movement in $\mathrm{T}$ cells showed that slowing the movement of microclusters toward the CSMAC prolongs signaling through the TCR (Nguyen et al. 2008). Thus, these observations support the proposal that the CSMAC forms a platform for internalization of immunoreceptors and attenuation of signaling (Lee et al. 2002). However, it seems that the division of labor between components of the IS may not be absolute since under certain conditions, such as stimulation with weak agonists, signaling in the cSMAC can be observed (Čemerski et al. 2008).

The role of the IS in antigen internalization is of particular significance in B cells, as this is known to be critical for the recruitment of $\mathrm{CD}^{+}{ }^{+}$T-helper cells required for maximal B-cell activation (Batista et al. 2001). It is clear that the BCR functions not only to mediate intracellular signaling, but also to target antigen to the correct compartment for intracellular processing (Aluvihare et al. 1997; Amigorena et al. 1994). Indeed, it has been shown that uptake through the BCR represents a more efficient means to accumulate antigen for presentation to T cells compared with fluid-phase endocytosis (Aluvihare et al. 1997; Cheng et al. 1999). BCR engagement initiates the reorganization of intracellular compartments to form a central multivesicular compartment enriched in MHC-II, antigen, and accessory molecules necessary for processing (Boes et al. 2004; Drake et al. 1999; Lankar et al. 2002; Siemasko and Clark 2001; Siemasko et al. 1998; Vascotto et al. 2007). Though the precise molecular requirements for the BCR-mediated 
Cytoskeleton Regulates B-cell Activation

internalization of antigen are not yet clear, actin-depolymerizing agents disrupt antigen internalization, establishing a critical role for the actin cytoskeleton during this step of Bcell activation (Barois et al. 1998; Brown and Song 2001). As B cells deficient in Syk show altered intracellular trafficking and impaired presentation of antigen to $\mathrm{CD} 4^{+} \mathrm{T}$ helper cells, it has been suggested that Syk plays a key role in regulating interactions between intracellular vesicles and actin filaments (Le Roux et al. 2007). In addition, the actin-based motor protein myosin II has been shown to be required for antigen processing and presentation (Vascotto et al. 2007). It has been put forward that most efficient BCR-mediated antigen internalization occurs when the actin cytoskeleton cooperates with clathrin- and membrane-raftmediated pathways (Stoddart et al. 2005). Although it is clear that the IS effectively coordinates the processes enabling antigen internalization, future imaging investigations will be vital to establish the molecular mechanisms underlying antigen internalization, presentation, and thus B-cell activation.

\section{CONCLUDING REMARKS}

It is evident from our discussion of the molecular events of B-cell activation that the actin cytoskeleton plays a key role at each stage, including: regulating the distribution of BCR in the resting cell membrane; mediating the assembly and stability of BCR microclusters; underlying the cellular spreading and contraction responses; and finally coordinating antigen internalization and processing. Thus, the B cell uses the actin cytoskeleton as a framework to successfully manage numerous molecular interactions. Interestingly, the cytoskeleton structure provides a scaffold to establish polarization of intracellular effectors and compartments, such as has been observed before cell division in $\mathrm{T}$ cells (Chang et al. 2007). This is a particularly intriguing issue in lymphocyte biology, as this type of polarization can lead to asymmetric segregation of components after cell division, potentially allowing generation of two daughter cells with different effector functions.

\section{ACKNOWLEDGMENTS}

Cancer Research UK supports research in the Lymphocyte Interaction Laboratory. We would like to thank members of the Lymphocyte Interaction Laboratory for critical reading of the manuscript particularly B. Treanor for providing the imaging data shown in Figure 1B.

\section{REFERENCES}

Ahearn J, Fischer M, Croix D, Goerg S, Ma M, Xia J, Zhou X, Howard R, Rothstein T, Carroll M. 1996. Disruption of the $\mathrm{Cr} 2$ locus results in a reduction in B-1a cells and in an impaired B cell response to T-dependent antigen. Immunity 4: 251-262.

Aluvihare VR, Khamlichi AA, Williams GT, Adorini L, Neuberger MS. 1997. Acceleration of intracellular targeting of antigen by the B-cell antigen receptor: Importance depends on the nature of the antigen-antibody interaction. EMBO J 16: 3553-3562.

Amigorena S, Drake JR, Webster P, Mellman I. 1994. Transient accumulation of new class II MHC molecules in a novel endocytic compartment in B lymphocytes. Nature 369: $113-120$.

Barois N, Forquet F, Davoust J. 1998. Actin microfilaments control the MHC class II antigen presentation pathway in B cells. J Cell Sci 111: 1791-1800.

Batista F, Neuberger M. 1998. Affinity dependence of the B cell response to antigen: A threshold, a ceiling, and the importance of off-rate. Immunity 8: 751-759.

Batista F, Neuberger M. 2000. B cells extract and present immobilized antigen: Implications for affinity discrimination. EMBO J 19: 513-520.

Batista F, Iber D, Neuberger M. 2001. B cells acquire antigen from target cells after synapse formation. Nature 411: 489-494.

Boes M, Cuvillier A, Ploegh H. 2004. Membrane specializations and endosome maturation in dendritic cells and $\mathrm{B}$ cells. Trends Cell Biol 14: 175-183.

Brooks S, Kirkham P, Freeberg L, Carter R. 2004. Binding of cytoplasmic proteins to the CD19 intracellular domain is high affinity, competitive, and multimeric. J Immunol 172: 7556-7564.

Brown BK, Song W. 2001. The actin cytoskeleton is required for the trafficking of the B cell antigen receptor to the late endosomes. Traffic 2: 414-427.

Bunnell S, Hong D, Kardon J, Yamazaki T, McGlade C, Barr V, Samelson L. 2002. T cell receptor ligation induces the formation of dynamically regulated signaling assemblies. J Cell Biol 158: 1263-1275.

Bunnell S, Kapoor V, Trible R, Zhang W, Samelson L. 2001. Dynamic actin polymerization drives $\mathrm{T}$ cell receptorinduced spreading: A role for the signal transduction adaptor LAT. Immunity 14: 315-329.

Byth K, Conroy L, Howlett S, Smith A, May J, Alexander D, Holmes N. 1996. CD45-null transgenic mice reveal a positive regulatory role for CD45 in early thymocyte 
N.E. Harwood and F.D. Batista

development, in the selection of CD4+CD8+ thymocytes, and B cell maturation. J Exp Med 183: 1707-1718.

Čemerski S, Das J, Giurisato E, Markiewicz MA, Allen PM, Chakraborty AK, Shaw AS. 2008. The balance between T cell receptor signaling and degradation at the center of the immunological synapse is determined by antigen quality. Immunity 29: 414-422.

Campi G, Varma R, Dustin M. 2005. Actin and agonist MHC-peptide complex-dependent $\mathrm{T}$ cell receptor microclusters as scaffolds for signaling. J Exp Med 202: 1031-1036.

Carrasco Y, Batista F. 2007. B cells acquire particulate antigen in a macrophage-rich area at the boundary between the follicle and the subcapsular sinus of the lymph node. Immunity 27: 160-171.

Carrasco Y, Fleire S, Cameron T, Dustin M, Batista F. 2004 LFA-1/ICAM-1 interaction lowers the threshold of B cell activation by facilitating B cell adhesion and synapse formation. Immunity 20: 589-599.

Chang JT, Palanivel VR, Kinjyo I, Schambach F, Intlekofer AM, Banerjee A, Longworth SA, Vinup KE, Mrass P, Oliaro J, et al. 2007. Asymmetric T lymphocyte division in the initiation of adaptive immune responses. Science 315: 1687-1691.

Chen L, Frank A, Adams J, Steinman R. 1978. Distribution of horseradish peroxidase (HRP)-anti-HRP immune complexes in mouse spleen with special reference to follicular dendritic cells. J Cell Biol 79: 184-199.

Cheng PC, Steele CR, Gu L, Song W, Pierce SK. 1999. MHC class II antigen processing in B cells: accelerated intracellular targeting of antigens. J Immunol 162: 7171-7180.

Choudhuri K, Wiseman D, Brown M, Gould K, van der Merwe P. 2005. T-cell receptor triggering is critically dependent on the dimensions of its peptide-MHC ligand. Nature 436: 578-582.

Conley ME, Dobbs AK, Farmer DM, Kilic S, Paris K, Grigoriadou S, Coustan-Smith E, Howard V, Campana D. 2009. Primary B cell immunodeficiencies: comparisons and contrasts. Annu Rev Immunol 27: 199-227.

Dal Porto J, Gauld S, Merrell K, Mills D, Pugh-Bernard A, Cambier J. 2004. B cell antigen receptor signaling 101. Mol Immunol 41: 599-613.

Davis S, van der Merwe P. 2006. The kinetic-segregation model: TCR triggering and beyond. Nat Immunol 7: 803-809.

Davis D, Chiu I, Fassett M, Cohen G, Mandelboim O, Strominger J. 1999. The human natural killer cell immune synapse. Proc Natl Acad Sci 96: 15062-15067.

DeFranco A. 1997. The complexity of signaling pathways activated by the BCR. Curr Opin Immunol 9: 296-308.

Depoil D, Fleire S, Treanor BL, Weber M, Harwood NE, Marchbank KL, Tybulewicz VL, Batista FD. 2008. CD19 is essential for B cell activation by promoting B cell receptor-antigen microcluster formation in response to membrane-bound ligand. Nat Immunol 9: 63-72.

DesMarais V, Ghosh M, Eddy R, Condeelis J. 2005. Cofilin takes the lead. J Cell Sci 118: 19-26.

Drake JR, Lewis TA, Condon KB, Mitchell RN, Webster P. 1999. Involvement of MIIC-like late endosomes in B cell receptor-mediated antigen processing in murine $\mathrm{B}$ cells. J Immunol 162: 1150-1155.
Engel P, Zhou L, Ord D, Sato S, Koller B, Tedder T. 1995. Abnormal B lymphocyte development, activation, and differentiation in mice that lack or overexpress the CD19 signal transduction molecule. Immunity 3: 39-50.

Faure S, Salazar-Fontana L, Semichon M, Tybulewicz V, Bismuth G, Trautmann A, Germain R, Delon J. 2004. ERM proteins regulate cytoskeleton relaxation promoting $\mathrm{T}$ cell-APC conjugation. Nat Immunol 5: 272-279.

Fearon D, Carroll M. 2000. Regulation of B lymphocyte responses to foreign and self-antigens by the CD19/ CD21 complex. Annu Rev Immunol 18: 393-422.

Fleire S, Goldman J, Carrasco Y, Weber M, Bray D, Batista F. 2006. B cell ligand discrimination through a spreading and contraction response. Science 312: 738-741.

Fournier E, Isakoff S, Ko K, Cardinale C, Inghirami G, Li Z, Curotto de Lafaille M, Skolnik E. 2003. The B cell SH2/ $\mathrm{PH}$ domain-containing adaptor Bam32/DAPP1 is required for T cell-independent II antigen responses. Curr Biol 13: 1858-1866.

Fujimoto M, Bradney A, Poe J, Steeber D, Tedder T. 1999. Modulation of B lymphocyte antigen receptor signal transduction by a CD19/CD22 regulatory loop. Immunity 11: 191-200.

Fujiwara T, Ritchie K, Murakoshi H, Jacobson K, Kusumi A. 2002. Phospholipids undergo hop diffusion in compartmentalized cell membrane. J Cell Biol 157: 1071-1081.

Garcia KC, Teyton L, Wilson IA. 1999. Structural basis of T cell recognition. Annu Rev Immunol 17: 369-397.

Gil D, Schamel WW, Montoya M, Sánchez-Madrid F, Alarcón B. 2002. Recruitment of Nck by CD3 epsilon reveals a ligand-induced conformational change essential for $\mathrm{T}$ cell receptor signaling and synapse formation. Cell 109: 901-912.

Gomez T, McCarney S, Carrizosa E, Labno C, Comiskey E, Nolz J, Zhu P, Freedman B, Clark M, Rawlings D, et al. 2006. HS1 functions as an essential actin-regulatory adaptor protein at the immune synapse. Immunity 24: 741-752.

Grakoui A, Bromley S, Sumen C, Davis M, Shaw A, Allen P, Dustin M. 1999. The immunological synapse: A molecular machine controlling $\mathrm{T}$ cell activation. Science 285: 221-227.

Gupta N, Wollscheid B, Watts J, Scheer B, Aebersold R, DeFranco A. 2006. Quantitative proteomic analysis of B cell lipid rafts reveals that ezrin regulates antigen receptor-mediated lipid raft dynamics. Nat Immunol 7: 625-633.

Halin C, Rodrigo Mora J, Sumen C, von Andrian U. 2005. In vivo imaging of lymphocyte trafficking. Annu Rev Cell Dev Biol 21: 581-603.

Han A, Saijo K, Mecklenbräuker I, Tarakhovsky A, Nussenzweig M. 2003. Bam32 links the B cell receptor to ERK and JNK and mediates B cell proliferation but not survival. Immunity 19: 621-632.

Hao J, Carey G, Zhan X. 2004. Syk-mediated tyrosine phosphorylation is required for the association of hematopoietic lineage cell-specific protein 1 with lipid rafts and B cell antigen receptor signalosome complex. J Biol Chem 279: 33413-33420. 
Harwood NE, Batista FD. 2008. New insights into the early molecular events underlying B cell activation. Immunity 28: 609-619.

Hennecke J, Wiley DC. 2001. T cell receptor-MHC interactions up close. Cell 104: 1-4.

Huang N, Han S, Hwang I, Kehrl J. 2005. B cells productively engage soluble antigen-pulsed dendritic cells: Visualization of live-cell dynamics of B cell-dendritic cell interactions. J Immunol 175: 7125-7134.

Ilani T, Vasiliver-Shamis G, Vardhana S, Bretscher A, Dustin ML. 2009. T cell antigen receptor signaling and immunological synapse stability require myosin IIA. Nat Immunol 10: $531-539$.

Irles C, Symons A, Michel F, Bakker T, van der Merwe P, Acuto O. 2003. CD45 ectodomain controls interaction with GEMs and Lck activity for optimal TCR signaling. Nat Immunol 4: 189-197.

Jaffe A, Hall A. 2005. Rho GTPases: Biochemistry and biology. Annu Rev Cell Dev Biol 21: 247-269.

Junt T, Scandella E, Ludewig B. 2008. Form follows function: Lymphoid tissue microarchitecture in antimicrobial immune defence. Nat Rev Immunol 8: 764-775.

Junt T, Moseman E, Iannacone M, Massberg S, Lang P, Boes M, Fink K, Henrickson S, Shayakhmetov D, Di Paolo N, et al. 2007. Subcapsular sinus macrophages in lymph nodes clear lymph-borne viruses and present them to antiviral B cells. Nature 450: 110-114.

Kaizuka Y, Douglass AD, Varma R, Dustin ML, Vale RD. 2007. Mechanisms for segregating $\mathrm{T}$ cell receptor and adhesion molecules during immunological synapse formation in Jurkat T cells. Proc Natl Acad Sci 104: 2029620301.

Kishihara K, Penninger J, Wallace V, Kündig T, Kawai K, Wakeham A, Timms E, Pfeffer K, Ohashi P, Thomas M. 1993. Normal B lymphocyte development but impaired $\mathrm{T}$ cell maturation in $\mathrm{CD} 45$-exon6 protein tyrosine phosphatase-deficient mice. Cell 74: 143-156.

Koppel E, Wieland C, van den Berg V, Litjens M, Florquin S, van Kooyk Y, van der Poll T, Geijtenbeek T. 2005. Specific ICAM-3 grabbing nonintegrin-related 1 (SIGNR1) expressed by marginal zone macrophages is essential for defense against pulmonary Streptococcus pneumoniae infection. Eur J Immunol 35: 2962-2969.

Krummel M, Sjaastad M, Wülfing C, Davis M. 2000. Differential clustering of $\mathrm{CD} 4$ and $\mathrm{CD} 3 \zeta$ during $\mathrm{T}$ cell recognition. Science 289: 1349-1352.

Kuhns MS, Davis MM, Garcia KC. 2006. Deconstructing the form and function of the TCR/CD3 complex. Immunity 24: $133-139$.

Kurosaki T. 1999. Genetic analysis of B cell antigen receptor signaling. Annu Rev Immunol 17: 555-592.

Kurosaki T. 2002. Regulation of B-cell signal transduction by adaptor proteins. Nat Rev Immunol 2: 354-363.

Kusumi A, Ike H, Nakada C, Murase K, Fujiwara T. 2005. Single-molecule tracking of membrane molecules: Plasma membrane compartmentalization and dynamic assembly of raft-philic signaling molecules. Semin Immunol 17: 3-21.

Lankar D, Vincent-Schneider H, Briken V, Yokozeki T, Raposo G, Bonnerot C. 2002. Dynamics of major histocompatibility complex class II compartments during B cell receptor-mediated cell activation. J Exp Med 195: 461-472.

Lanzavecchia A. 1985. Antigen-specific interaction between T and B cells. Nature 314: 537-539.

Le Roux D, Lankar D, Yuseff M-I, Vascotto F, Yokozeki T, Faure-André G, Mougneau E, Glaichenhaus N, Manoury B, Bonnerot C, et al. 2007. Syk-dependent actin dynamics regulate endocytic trafficking and processing of antigens internalized through the B-cell receptor. Mol Biol Cell 18: 3451-3462.

Lee K, Holdorf A, Dustin M, Chan A, Allen P, Shaw A. 2002. $T$ cell receptor signaling precedes immunological synapse formation. Science 295: 1539-1542.

Li X, Sandoval D, Freeberg L, Carter R. 1997. Role of CD19 tyrosine 391 in synergistic activation of B lymphocytes by coligation of CD19 and membrane Ig. J Immunol 158: 5649-5657.

Lillemeier BF, Pfeiffer JR, Surviladze Z, Wilson BS, Davis MM. 2006. Plasma membrane-associated proteins are clustered into islands attached to the cytoskeleton. Proc Natl Acad Sci 103: 18992-18997.

MacLennan I. 1994. Germinal centers. Annu Rev Immunol 12: $117-139$.

MacLennan IC, Toellner KM, Cunningham AF, Serre K, Sze DM, Zúñiga E, Cook MC, Vinuesa CG. 2003. Extrafollicular antibody responses. Immunol Rev 194: 8-18.

Mandel T, Phipps R, Abbot A, Tew J. 1980. The follicular dendritic cell: long term antigen retention during immunity. Immunol Rev 53: 29-59.

Marshall A, Niiro H, Lerner C, Yun T, Thomas S, Disteche C, Clark E. 2000. A novel B lymphocyte-associated adaptor protein, Bam32, regulates antigen receptor signaling downstream of phosphatidylinositol 3-kinase. J Exp Med 191: 1319-1332.

Monks C, Freiberg B, Kupfer H, Sciaky N, Kupfer A. 1998. Three-dimensional segregation of supramolecular activation clusters in T cells. Nature 395: 82-86.

Mustelin T, Coggeshall K, Altman A. 1989. Rapid activation of the T-cell tyrosine protein kinase pp56lck by the CD45 phosphotyrosine phosphatase. Proc Natl Acad Sci 86: 6302-6306.

Nguyen K, Sylvain NR, Bunnell SC. 2008. T cell costimulation via the integrin VLA-4 inhibits the actin-dependent centralization of signaling microclusters containing the adaptor SLP-76. Immunity 28: 810-821.

Niiro H, Allam A, Stoddart A, Brodsky F, Marshall A, Clark E. 2004. The B lymphocyte adaptor molecule of 32 kilodaltons (Bam32) regulates B cell antigen receptor internalization. J Immunol 173: 5601-5609.

Phan T, Grigorova I, Okada T, Cyster J. 2007. Subcapsular encounter and complement-dependent transport of immune complexes by lymph node B cells. Nat Immunol 8: $992-1000$

Phan TG, Green JA, Gray EE, Xu Y, Cyster JG. 2009. Immune complex relay by subcapsular sinus macrophages and noncognate B cells drives antibody affinity maturation. Nat Immunol 10: 786-793.

Potter T, Grebe K, Freiberg B, Kupfer A. 2001. Formation of supramolecular activation clusters on fresh ex vivo $\mathrm{CD} 8+\mathrm{T}$ cells after engagement of the $\mathrm{T}$ cell antigen 
N.E. Harwood and F.D. Batista

receptor and CD8 by antigen-presenting cells. Proc Natl Acad Sci 98: 12624-12629.

Qi H, Egen JG, Huang AY, Germain RN. 2006. Extrafollicular activation of lymph node B cells by antigen-bearing dendritic cells. Science 312: 1672-1676.

Rajewsky K. 1996. Clonal selection and learning in the antibody system. Nature 381: 751-758.

Randall K, Lambe T, Johnson A, Kucharska E, Domaschenz H, Whittle B, Tze L, Enders A, Crockford T, BouriezJones T, et al. 2009. Dock8 mutations cripple B cell immunological synapses, germinal centers and longlived antibody production. Nat Immunol 10: 1283-1291.

Reth M. 1989. Antigen receptor tail clue. Nature 338: $383-$ 384.

Rickert R, Rajewsky K, Roes J. 1995. Impairment of Tcell-dependent B-cell responses and B-1 cell development in CD19-deficient mice. Nature 376: 352-355.

Rock K, Benacerraf B, Abbas A. 1984. Antigen presentation by hapten-specific B lymphocytes. I. Role of surface immunoglobulin receptors. J Exp Med 160: 1102-1113.

Rolli V, Gallwitz M, Wossning T, Flemming A, Schamel W, Zürn C, Reth M. 2002. Amplification of B cell antigen receptor signaling by a Syk/ITAM positive feedback loop. Mol Cell 10: 1057-1069.

Rudolph MG, Stanfield RL, Wilson IA. 2006. How TCRs bind MHCs, peptides, and coreceptors. Annu Rev Immunol 24: 419-466.

Sako Y, Kusumi A. 1994. Compartmentalized structure of the plasma membrane for receptor movements as revealed by a nanometer-level motion analysis. J Cell Biol 125: 1251-1264.

Sato S, Miller A, Howard M, Tedder T. 1997. Regulation of B lymphocyte development and activation by the CD19/ CD21/CD81/Leu 13 complex requires the cytoplasmic domain of CD19. J Immunol 159: 3278-3287.

Schamel W, Reth M. 2000. Monomeric and oligomeric complexes of the B cell antigen receptor. Immunity 13: 5-14.

Schamel WW, Risueño RM, Minguet S, Ortíz AR, Alarcón B 2006. A conformation- and avidity-based proofreading mechanism for the TCR-CD3 complex. Trends Immunol 27: $176-182$.

Scharenberg A, Humphries L, Rawlings D. 2007. Calcium signalling and cell-fate choice in B cells. Nat Rev Immunol 7: 778-789.

Schreiner GF, Braun J, Unanue ER. 1976. Spontaneous redistribution of surface immunoglobulin in the motile B lymphocyte. J Exp Med 144: 1683-1688.

Secrist JP, Burns LA, Karnitz L, Koretzky GA, Abraham RT. 1993. Stimulatory effects of the protein tyrosine phosphatase inhibitor, pervanadate, on T-cell activation events. J Biol Chem 268: 5886-5893.

Shinohara H, Kurosaki T. 2006. Genetic analysis of B cell signaling. Subcell Biochem 40: 145-187.

Siemasko K, Clark MR. 2001. The control and facilitation of MHC class II antigen processing by the BCR. Curr Opin Immunol 13: 32-36.

Siemasko K, Eisfelder BJ, Williamson E, Kabak S, Clark MR. 1998. Cutting edge: Signals from the B lymphocyte antigen receptor regulate MHC class II containing late endosomes. J Immunol 160: 5203-5208.
Simons K, Ikonen E. 1997. Functional rafts in cell membranes. Nature 387: 569-572.

Simson R, Sheets ED, Jacobson K. 1995. Detection of temporary lateral confinement of membrane proteins using single-particle tracking analysis. Biophys J 69: 989-993.

Singer SJ, Nicolson GL. 1972. The fluid mosaic model of the structure of cell membranes. Science 175: 720-731.

Sohn H, Pierce S, Tzeng S. 2008. Live cell imaging reveals that the inhibitory Fc\{gamma\}RIIB destabilizes B cell receptor membrane-lipid interactions and blocks immune synapse formation. J Immunol 180: 793-799.

Stackpole CW, Jacobson JB, Lardis MP. 1974. Two distinct types of capping of surface receptors on mouse lymphoid cells. Nature 248: 232-234.

Stinchcombe J, Bossi G, Booth S, Griffiths G. 2001. The immunological synapse of CTL contains a secretory domain and membrane bridges. Immunity 15: 751-761.

Stoddart A, Jackson AP, Brodsky FM. 2005. Plasticity of B cell receptor internalization upon conditional depletion of clathrin. Mol Biol Cell 16: 2339-2348.

Suzuki K, Grigorova I, Phan TG, Kelly LM, Cyster JG. 2009. Visualizing B cell capture of cognate antigen from follicular dendritic cells. J Exp Med 206: 1485-1493.

Tew JG, Phipps RP, Mandel TE. 1980. The maintenance and regulation of the humoral immune response: Persisting antigen and the role of follicular antigen-binding dendritic cells as accessory cells. Immunol Rev 53: 175201.

Tolar P, Sohn H, Pierce S. 2005. The initiation of antigeninduced B cell antigen receptor signaling viewed in living cells by fluorescence resonance energy transfer. Nat Immunol 6: 1168-1176.

Tolar P, Hanna J, Krueger PD, Pierce SK. 2009. The constant region of the membrane immunoglobulin mediates $\mathrm{B}$ cell-receptor clustering and signaling in response to membrane antigens. Immunity 30: 44-55.

Treanor B, Batista F. 2007. Mechanistic insight into lymphocyte activation through quantitative imaging and theoretical modelling. Curr Opin Immunol 19: 476-483.

Treanor B, Depoil D, Gonzalez-Granja A, Barral P, Weber M, Dushek O, Bruckbauer A, Batista FD. 2010. The membrane skeleton controls diffusion dynamics and signaling through the B cell receptor. Immunity 32: 187-199.

Tuveson D, Carter R, Soltoff S, Fearon D. 1993. CD19 of B cells as a surrogate kinase insert region to bind phosphatidylinositol 3-kinase. Science 260: 986-989.

Unanue ER, Perkins WD, Karnovsky MJ. 1972. Ligandinduced movement of lymphocyte membrane macromolecules. I. Analysis by immunofluorescence and ultrastructural radioautography. J Exp Med 136: 885-906.

van der Merwe PA. 2001. The TCR triggering puzzle. Immunity 14: 665-668.

Vascotto F, Lankar D, Faure-André G, Vargas P, Diaz J, Le Roux D, Yuseff M-I, Sibarita J-B, Boes M, Raposo G, et al. 2007. The actin-based motor protein myosin II regulates MHC class II trafficking and BCR-driven antigen presentation. J Cell Biol 176: 1007-1019.

Weber M, Treanor B, Depoil D, Shinohara H, Harwood NE, Hikida M, Kurosaki T, Batista FD. 2008. Phospholipase C- $\gamma 2$ and Vav cooperate within signaling microclusters 
to propagate $B$ cell spreading in response to membranebound antigen. J Exp Med 205: 853-868.

Wykes M, Pombo A, Jenkins C, MacPherson G. 1998. Dendritic cells interact directly with naive B lymphocytes to transfer antigen and initiate class switching in a primary T-dependent response. J Immunol 161: 1313-1319.

Xu C, Gagnon E, Call ME, Schnell JR, Schwieters CD, Carman CV, Chou JJ, Wucherpfennig KW. 2008. Regulation of T cell receptor activation by dynamic membrane binding of the CD3epsilon cytoplasmic tyrosine-based motif. Cell 135: 702-713.

Yamanashi Y, Fukuda T, Nishizumi H, Inazu T, Higashi K, Kitamura D, Ishida T, Yamamura H, Watanabe T, Yamamoto T. 1997. Role of tyrosine phosphorylation of HS1 in B cell antigen receptor-mediated apoptosis. $J$ Exp Med 185: 1387-1392.
Yokosuka T, Sakata-Sogawa K, Kobayashi W, Hiroshima M Hashimoto-Tane A, Tokunaga M, Dustin M, Saito T 2005. Newly generated T cell receptor microclusters initiate and sustain $\mathrm{T}$ cell activation by recruitment of Zap70 and SLP-76. Nat Immunol 6: 1253-1262.

Yonezawa N, Homma Y, Yahara I, Sakai H, Nishida E. 1991. A short sequence responsible for both phosphoinositide binding and actin binding activities of cofilin. J Biol Chem 266: $17218-17221$.

Zhang W, Samelson L. 2000. The role of membrane-associated adaptors in T cell receptor signalling. Semin Immunol 12: 35-41.

Zhu J, Brdicka T, Katsumoto T, Lin J, Weiss A. 2008. Structurally distinct phosphatases CD45 and CD148 both regulate $\mathrm{B}$ cell and macrophage immunoreceptor signaling. Immunity 28: 183-196. 


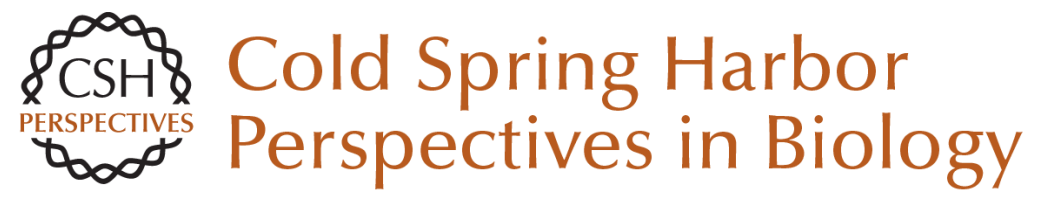

\title{
The Cytoskeleton Coordinates the Early Events of B-cell Activation
}

\author{
Naomi E. Harwood and Facundo D. Batista
}

Cold Spring Harb Perspect Biol 2011; doi: 10.1101/cshperspect.a002360 originally published online November 3, 2010

\section{Subject Collection Immunoreceptor Signaling}

The Coordination of T-cell Function by Serine/Threonine Kinases

David Finlay and Doreen Cantrell

ITAM-mediated Signaling by the T-Cell Antigen Receptor

Paul E. Love and Sandra M. Hayes

Coordination of Receptor Signaling in Multiple Hematopoietic Cell Lineages by the Adaptor

Protein SLP-76

Martha S. Jordan and Gary A. Koretzky

The Cytoskeleton Coordinates the Early Events of B-cell Activation

Naomi E. Harwood and Facundo D. Batista

An Enigmatic Tail of CD28 Signaling

Jonathan S. Boomer and Jonathan M. Green

\section{Mediation of T-Cell Activation by Actin Meshworks}

Peter Beemiller and Matthew F. Krummel

T-Cell Signaling Regulated by the Tec Family

Kinase, Itk

Amy H. Andreotti, Pamela L. Schwartzberg, Raji E. Joseph, et al.
Perspectives for Computer Modeling in the Study of T Cell Activation Jesse Coward, Ronald N. Germain and Grégoire Altan-Bonnet

Structural Biology of the T-cell Receptor: Insights into Receptor Assembly, Ligand Recognition, and Initiation of Signaling

Kai W. Wucherpfennig, Etienne Gagnon, Melissa J. Call, et al.

Src-family and Syk Kinases in Activating and

Inhibitory Pathways in Innate Immune Cells:

Signaling Cross Talk Clifford A. Lowell

The LAT Story: A Tale of Cooperativity, Coordination, and Choreography

Lakshmi Balagopalan, Nathan P. Coussens, Eilon Sherman, et al.

Antigen Receptor Signaling to NF- $\mathrm{KB}$ via CARMA1, BCL10, and MALT1 Margot Thome, Jean Enno Charton, Christiane Pelzer, et al.

It's All About Change: The Antigen-driven Initiation of B-Cell Receptor Signaling Wanli Liu, Hae Won Sohn, Pavel Tolar, et al.

ZAP-70: An Essential Kinase in T-cell Signaling Haopeng Wang, Theresa A. Kadlecek, Byron B. Au-Yeung, et al.

For additional articles in this collection, see http://cshperspectives.cshlp.org/cgi/collection/

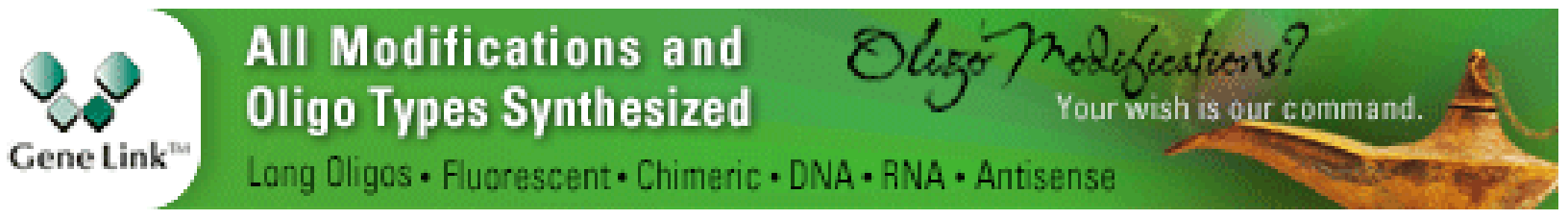




\section{Lipid Signaling in T-Cell Development and Function \\ Yina H. Huang and Karsten Sauer}

Understanding the Structure and Function of the Immunological Synapse

Michael L. Dustin, Arup K. Chakraborty and Andrey S. Shaw

For additional articles in this collection, see http://cshperspectives.cshlp.org/cgi/collection/

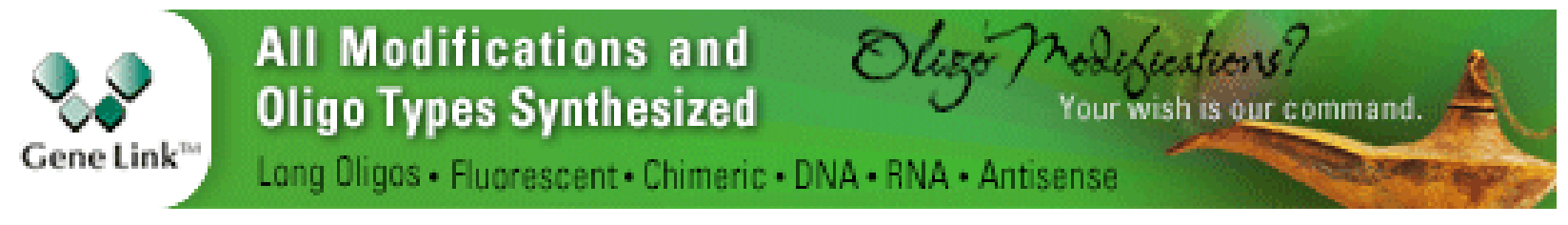

Copyright @ 2011 Cold Spring Harbor Laboratory Press; all rights reserved 